Mathematical Research Letters 8, 729-744 (2001)

\title{
CRITICAL EXPONENTS FOR TWO-DIMENSIONAL PERCOLATION
}

\author{
Stanislav Smirnov and Wendelin Werner
}

\begin{abstract}
We show how to combine Kesten's scaling relations, the determination of critical exponents associated to the stochastic Loewner evolution process by Lawler, Schramm, and Werner, and Smirnov's proof of Cardy's formula, in order to determine the existence and value of critical exponents associated to percolation on the triangular lattice.
\end{abstract}

\section{Introduction}

The goal of the present note is to review and clarify the consequences of recent papers and preprints concerning the existence and values of critical exponents for site percolation on the triangular lattice.

Suppose that $p \in(0,1)$ is fixed. Each vertex of the triangular lattice (or equivalently each hexagon in the honeycomb lattice) is open (or colored blue) with probability $p$ and closed (or colored yellow) with probability $1-p$, independently of each other. It is now well-known (and due to Kesten and Wierman, see the textbooks $[12,10])$ that when $p \leq 1 / 2$, there is almost surely no infinite cluster of open vertices, while if $p>1 / 2$, there is a.s. a unique such infinite cluster and the probability $\theta(p)$ that the origin belongs to this infinite cluster is then positive. Arguments from theoretical physics predicted that when $p$ approaches the critical value $1 / 2$ from above, $\theta(p)$ behaves roughly like $(p-1 / 2)^{5 / 36}$. This number $5 / 36$ is one of several critical exponents that are supposed to be independent of the considered planar lattice and that are describing the behaviour of percolation near its critical point $p=p_{c}\left(p_{c}=1 / 2\right.$ for this particular model). Our goal in the present paper is to point out that this result, as well as other related statements, is a consequence of the combination of various papers:

- In [13], Kesten has shown that in order to understand the behaviour of percolation near its critical point (and in particular existence and values of certain critical exponents), it is sufficient to study what happens at the critical point i.e. here when $p=p_{c}=1 / 2$. In particular, many results would follow from the existence and the values of the exponents describing the decay when $R$ goes to infinity of the probabilities (at $p=1 / 2$ ) of the events $A_{R}^{1}$ and $A_{R}^{2}$ that if we restrict the percolation to the disc of radius $R$, there exist one (respectively two disjoint) blue clusters joining the vicinity

Received September 20, 2001.

2000 Mathematics Subject Classification. 60K35, 82B27, 82B43. 
of the origin (say that are at distance less than two of the origin) to the circle of radius $R$.

- In [28], Schramm defined a family of random evolution processes based on Loewner's equation, and pointed out that one of them (the stochastic Loewner evolution process with parameter 6, referred to as $S L E_{6}$ in the sequel) is the only possible conformally invariant scaling limit of discrete critical percolation cluster interfaces. In a series of papers $[15,16,17,21]$, Lawler, Schramm, and Werner have derived various properties of $S L E_{6}$ (relation between radial and chordal processes, locality property etc), computed critical exponents associated to $S L E_{6}$, and then used these exponents to determine the value of Brownian exponents (for instance, the Hausdorff dimension of the planar Brownian frontier is a.s. 4/3).

- In [30, 31], Smirnov proved that indeed, critical site percolation on the triangular lattice has a conformally invariant scaling limit when the mesh of the lattice tends to zero, and in particular, that the discrete cluster interfaces converge to this stochastic Loewner evolution process.

In the present paper, we will outline how one can combine all these results to show that the critical exponents for discrete site percolation on the triangular lattice are those predicted in the physics literature. All the results that we prove in the present paper have been conjectured (on the basis of numerics and heuristics) and predicted (using Coulomb Gas methods, Conformal Field Theory, or Quantum Gravity) by physicists. See e.g., Den Nijs [8], Nienhuis et al. [22, 23], Pearson [24], Cardy [5], Sapoval-Rosso-Gouyet [27], Grossman-Aharony [11], Duplantier-Saleur [26], Cardy [6], Duplantier [9], Aizenman-DuplantierAharony [2] and the references in these papers.

There exists a vast theoretical physics literature on this subject and we do not claim that this list covers all important contributions to it.

Note the description of the scaling limit via $S L E_{6}$ enables also to derive some results that have not appeared in the physics literature. For instance, an analogue of Cardy's formula "in the bulk" [29], or a description of the so-called backbone exponent [21].

\section{Kesten's scaling relations}

Let us now introduce some notation. Denote by $N$ the cardinality of the cluster $C$ containing the origin. Recall that

$$
\theta(p)=\mathbf{P}_{p}[N=\infty]
$$

where $\mathbf{P}_{p}, \mathbf{E}_{p}$ corresponds to site percolation on the triangular lattice with parameter $p$. Let

$$
\chi(p)=\mathbf{E}_{p}\left[N 1_{N<\infty}\right] .
$$


This corresponds to the average cardinality of finite clusters. Let

$$
\xi(p)=\left[\frac{\mathbf{E}_{p}\left[\sum_{y \in C}|y|^{2} 1_{N<\infty}\right]}{\chi(p)}\right]^{1 / 2} .
$$

This is the so-called correlation length corresponding to the "typical radius" of a finite cluster. Other definitions of correlation length are possible, see e.g., $[10,13,7]$; for instance, define $\xi^{*}(p)$ by the relation

$\mathbf{P}[0$ is connected to $x$ by a finite cluster $]=\exp \left\{-x / \xi^{*}(p)+o(x)\right\}$ when $x \rightarrow+\infty$.

If $x$ tends to infinity along some fixed direction, existence of $\xi^{*}$ (dependent on the chosen direction) easily follows. So to make the definition rigorous, one can assume for instance that $x \in \mathbb{R}$. On the other hand, the proof shows that the asymptotic behaviour of $\xi^{*}(p)$ in the neighbourhood of $p_{c}=1 / 2$ is independent of the chosen direction (i.e., (iv) holds for all given directions).

Theorem 1 (Behaviour near the critical point).

- (i) When $p \rightarrow 1 / 2+$,

$$
\theta(p)=(p-1 / 2)^{5 / 36+o(1)} .
$$

- (ii) When $p \rightarrow 1 / 2$

$$
\chi(p)=(p-1 / 2)^{-43 / 18+o(1)} .
$$

- (iii) When $p \rightarrow 1 / 2$,

$$
\xi(p)=(p-1 / 2)^{-4 / 3+o(1)} .
$$

- (iv) When $p \rightarrow 1 / 2$,

$$
\xi^{*}(p)=(p-1 / 2)^{-4 / 3+o(1)} .
$$

It has been shown by Kesten in [13] (using also the remark following Lemma 8 in [13]; it can be shown that (iv) holds once (iii) holds, using the estimates in [13], see also section 3 in [7]) that all these results hold provided that: When $p=1 / 2$ and $R \rightarrow \infty$,

$$
\mathbf{P}\left[A_{R}^{1}\right]=R^{-5 / 48+o(1)}
$$

and

$$
\mathbf{P}\left[A_{R}^{2}\right]=R^{-5 / 4+o(1)} .
$$

The relations between these two critical exponents and those appearing in Theorem 1 are sometimes known as scaling relations.

Relation (1) is proved in [21], combining the computation of an exponent for $S L E_{6}$ with the results of $[30,31]$. In the rest of this paper, we fix $p=1 / 2$ and we shall see how (2) and other closely related results (we will also briefly discuss (1)) follow from the combination of $[30,31]$ with $[15,16,17,21]$. Independently, 
Yu Zhang [33] has recently anounced a proof of (2) and of (iii) in the above Theorem, probably using similar thoughts and arguments as those that we shall present here.

\section{Half-plane exponents}

In this section, we are going to study the decay when $R \rightarrow \infty$, of the probability of the events that there exist $j$ disjoint blue paths that stay in the upper half-plane, start at the vicinity of the origin, and reach distance $R$. Besides being of independent interest, this section serves as a model for the determination of exponents in the plane.

To be more precise, consider critical percolation with fixed mesh equal to 1 , and consider the event $G_{j}(r, R)$ that there exists $j$ disjoint blue crossings of the semi-annulus $A_{+}(r, R):=\{z: r<|z|<R, \Im(z)>0\}$. By a blue crossing we mean a (discrete) simple blue curve, i.e. a sequence of distinct blue hexagons connecting the "semicircle" boundaries of $A_{+}(r, R)$, where consecutive hexagons are adjacent.

Actually, we slightly modify the definition of $A_{+}(r, R)$ as follows: All hexagons intersecting the circle of radius $r$ will be in $A_{+}(r, R)$ while those which intersect the circle of radius $R$ are not in $A_{+}(r, R)$. A crossing of $A_{+}(r, R)$ is then a bluse simple curve from $|z|=r$ to a hexagon on the "outer boundary" of $A_{+}(r, R)$.

One could as well take a semi-hexagonal or a triangular shape instead of the semi-circles to simplify the discrete approximation. Define $a_{j}(r, R):=$ $\mathbf{P}\left[G_{j}(r, R)\right]$.

Remark 2 (Changing the colors). The convention to study blue crossings is not restrictive: it is standard (see e.g., $[2,15])$ that for any given sequence of colours, the probability that there exist left-right crossings of a topological rectangle (for discrete critical percolation) of some prescribed colours in prescribed order is in fact independent of this sequence of colours. It follows that this probability is comparable (for long rectangles) to the probability of $j$ crossings of arbitrary colours. One can also check that the probabilities of at least $j$ crossings, and of exactly $j$ crossings are comparable. The reason is that one can explore the crossings from "below" one-by-one and flip all colours above some of them without changing the probabilities of configurations.

We prove the following result, which was predicted by physicists:

Theorem 3 (Half-plane exponents). For any $j \geq 1$, and for all large enough $r$ (i.e. $r>\operatorname{const}(j))$,

$$
a_{j}(r, R)=R^{-j(j+1) / 6+o(1)} \text { when } R \rightarrow \infty .
$$

We first show that the theorem is a direct consequence of the following two facts, which will be discussed in the remainder of the section:

- Identification between $S L E_{6}$ and continuum percolation implies that exponents for continuum percolation are equal to the exponents for $S L E_{6}$, computed in [15]. This can be written in terms of discrete percolation: 


$$
\lim _{\rho \rightarrow \infty} a_{j}(\rho, R \rho)=R^{-j(j+1) / 6+o(1)} \text { when } R \rightarrow \infty .
$$

- Crossing probabilities enjoy the following (approximate) multiplicativity property with some positive $c=\operatorname{const}(j)$, provided $r^{\prime \prime} \geq r^{\prime} \geq r>j$ (cf. $[13,14])$ :

$$
a_{j}\left(r, r^{\prime}\right) a_{j}\left(r^{\prime}, r^{\prime \prime}\right) \geq a_{j}\left(r, r^{\prime \prime}\right) \geq c a_{j}\left(r, r^{\prime}\right) a_{j}\left(r^{\prime}, r^{\prime \prime}\right) .
$$

In fact, as $a_{j}(r, R)$ is decreasing in $R$, to establish Theorem 3 it is sufficient to show that for any fixed $\epsilon$, there exists $K>1$ such that for sufficiently large $n$,

$$
\left(K^{n}\right)^{-j(j+1) / 6-\epsilon} \leq a_{j}\left(r, r K^{n}\right) \leq\left(K^{n}\right)^{-j(j+1) / 6+\epsilon} .
$$

To prove (5), we use (3) and choose large enough $K$ so that

$$
c^{-1} K^{-j(j+1) / 6-\epsilon / 2} \leq a_{j}(\rho, \rho K) \leq K^{-j(j+1) / 6+\epsilon / 2},
$$

for sufficiently large $\rho$. Together with (4) this implies

$$
\begin{array}{r}
\text { const } \prod_{m=1}^{n} K^{-j(j+1) / 6-\epsilon / 2} \leq \prod_{m=1}^{n} c a_{j}\left(K^{m-1} r, K^{m} r\right) \leq a_{j}\left(r, r K^{n}\right) \leq \\
\leq \prod_{m=1}^{n} a_{j}\left(K^{m-1} r, K^{m} r\right) \leq \mathrm{const} \prod_{m=1}^{n} K^{-j(j+1) / 6+\epsilon / 2}
\end{array}
$$

and (5) readily follows.

To prove Theorem 3 it remains to check (3) and (4).

\subsection{Chordal processes.}

Chordal exploration process. Suppose for a moment that $\Omega$ is a simply connected set of hexagons, and that $a$ and $b$ are two distinct vertices (of the honeycombe lattice) that are on its boundary. For convenience, we will often identify an arbitrary domain $\Omega$ with its discrete hexagonal approximation. Colour all hexagons on the boundary of $\Omega$ that are between $a$ and $b$ in counter-clockwise order (resp. clockwise order) in blue (resp. yellow) and call this set of blue hexagons $\partial_{b}$ (resp. $\partial_{y}$ ). There exists a unique curve separating the blue cluster attached to $\partial_{b}$ from the yellow cluster attached to $\partial_{y}$. This is the exploration process from $a$ to $b$ in $\Omega$. Note that this curve is a simple curve that has blue hexagons to its left and yellow hexagons to its right (if seen from $a$ to $b$ ), and that it can be defined dynamically, as an "exploration process" that turns right when it meets a yellow hexagon and left when it meets a blue hexagon. In particular, for a fixed curve $\gamma$, the event that the exploration process is equal to $\gamma$ depends only on the state of the hexagons that are in the neighbourhood of $\gamma$. 
By Remark 2, $a_{j}(r, R)$ is also the probability of the event of $j$ crossings of alternate colors, which is can be described as discrete exploration process inside the semiannulus $A_{+}(r, R)$ from the point $r$ (i.e., the real point $r$ in the complex plane) to the point $-R$ making $j$ crossings before hitting the interval $[-R,-r]$. Note that for odd $j$ this can also be described as the existence of $(j-1) / 2$ disjoint yellow clusters that cross the semi-annulus.

Chordal $S L E_{6}$. One can view chordal $S L E_{6}$ as follows (e.g., [15, 25]): For any simply connected open set $\Omega \subset \mathbb{C}, \Omega \neq \mathbb{C}$ and two distinguished points (or ends) $a$ and $b$ on its boundary, it is a random continuous curve $\gamma$ from $a$ to $b$ in $\bar{\Omega}$. The law of this curve is conformally invariant by construction, this curve has double-points but no "self-crossings," and is of fractal dimension $7 / 4$ almost surely $([25,3])$.

Critical exponents associated to the $S L E_{6}$ curve in a semi-annulus have been computed in $[15,17]$ : Define the probability $a_{j}^{\text {sle }}(r, R)$ that $S L E_{6}$ from $r$ to $-R$ in the semi-annulus $A_{+}(r, R)$ makes $j$ crossings before its hitting time $\tau$ of the interval $[-R,-r]$. Note also that because of the conformal invariance, $a_{j}^{\text {sle }}(r, R)$ depends on the ratio $R / r$ only: $a_{j}^{\text {sle }}(r, R)=a_{j}^{\text {sle }}(R / r)$.

Let $\sigma$ denote the time at which $S L E_{6}$ performs its first crossing of the semiannulus. At this time, the $S L E_{6}$ has still to perform $j-1$ crossings between the two circles in the connected component $U$ of $A_{+}(r, R) \backslash \gamma[0, \sigma]$ before $\tau$. By conformal invariance and the strong Markov property of $S L E_{6}$ at time $\sigma$, it follows that given $\gamma[0, \sigma]$, the conditional probability that $S L E_{6}$ makes the $j$ crossings is $a_{j-1}^{\text {sle }}(S)$ where $S / \pi$ is the extremal distance between the two circles in $U$. Hence,

$$
a_{j}^{s l e}(R / r)=\mathbf{E}\left[1_{\sigma<\tau} a_{j-1}(S)\right] .
$$

Theorem 2.6 in [15] states that that for all non-negative $\lambda$,

$$
\mathbf{E}\left[1_{\sigma<\tau} S^{-\lambda}\right] \asymp R^{-u(\lambda)} \text { when } R \rightarrow \infty,
$$

where $u(\lambda)=(6 \lambda+1+\sqrt{24 \lambda+1}) / 6$, and $\asymp$ means that the ratio between both quantities remain bounded and bounded away from zero. As $u^{\circ j}(0)=j(j+1) / 6$, it therefore follows by induction that for all $j \geq 1$,

$$
a_{j}^{\text {sle }}(R) \asymp R^{-j(j+1) / 6}, \text { when } R \rightarrow \infty .
$$

Exploration process and $S L E_{6}$. In [30] it is shown among other things that the discrete exploration process from $a$ to $b$ in the discretized approximation of $\Omega$ converges in law towards $S L E_{6}$, with respect to the Hausdorff topology on simple curves, when the mesh of the lattice goes to zero. In fact [31], it is also possible to derive a slightly stronger statement that loosely speaking the convergence takes place uniformly with respect to the domain $\Omega$ and the location of the endpoints $a$ and $b$.

Hence it follows from [30], that when the mesh of the lattice goes to zero, the probability that the discrete exploration process makes $j$ crossings of a semiannulus converges to that for $S L E_{6}$. Alternatively we can increase the domain while preserving the mesh, and conclude that 


$$
\lim _{\rho \rightarrow \infty} a_{j}(\rho r, \rho R)=a_{j}^{s l e}(r, R) .
$$

Let us stress that this uses the following "a priori bounds" (cf. [21, 13, 14]): For some fixed $r$ and $\epsilon>0$ and for all sufficiently large $R$,

$$
a_{3}(r, R) \leq R^{-1-\epsilon}
$$

as well as an analogous result for 6 arms in the plane (see (11) in the next section). These bounds for instance prevent the possibility that with positive probability, a crossing of the rectangle appears in the scaling limit while it was not present in the discrete case, or that two distinct crossings collapse into one in the scaling limit (see [31] for more details).

Equations (6) and (7) clearly imply the desired (3).

3.2. Multiplicativity. The left inequality in the "approximate multiplicativity" (4) is immediate since $A_{+}\left(r, r^{\prime}\right)$ and $A_{+}\left(r^{\prime}, r^{\prime \prime}\right)$ are disjoint.

The right inequality is elementary using an argument involving the RussoSeymour-Welsh theory (referred to as RSW theory in the sequel). If $2 r<r^{\prime}<$ $r^{\prime \prime} / 2$, one can use the Harris-FKG (Fortuin-Kasteleyn-Ginibre) inequality and the events that there exist $\mathrm{j}$ disjoint blue crossings of $A_{+}\left(r^{\prime} / 2,2 r^{\prime}\right)$, and also $j$ disjoint blue crossings of the "long side" of the semi-annuli $A_{+}\left(r^{\prime} / 2, r^{\prime}\right)$ (i.e. that join the two real segments) and $\left.A_{+}\left(r^{\prime}, r^{\prime} / 2\right)\right)$. This argument relies on the fact that all crossings have the same colour. As we shall see later, things are more involved for plane exponents because of the fact that we will be considering crossings of the two colours.

This is the only case needed in the proof, but remaining cases are also easy: if e.g. $r^{\prime} \geq r^{\prime \prime} / 2$ then by the reasoning above and standard RSW theory,

$$
a_{j}\left(r, r^{\prime \prime}\right) \geq a_{j}\left(r, 2 r^{\prime}\right) \geq c a_{j}\left(r, r^{\prime}\right) a_{j}\left(r^{\prime}, 2 r^{\prime}\right) \geq c^{\prime} a_{j}\left(r, r^{\prime}\right) a_{j}\left(r^{\prime}, r^{\prime \prime}\right) .
$$

\section{Plane exponents}

We now study the plane exponents, i.e. probabilities of crossings of annuli instead of semi-annuli. There is a profound difference with the half-plane case, when we had a "starting half-line," which allowed to choose for a given configuration a "canonical realization" of crossings (the lowest, the second from below, and so on) and to change colours of crossings at will.

In contrast, when one studies $j$ crossings of an annulus of the same colour, then there is no canonical way to choose their realization. There was a prediction by theoretical physicists in the case $j=1$ (this is the "one-arm exponent" that we will discuss later), but not for $j \geq 2$. However, conformal invariance and $S L E_{6}$ can be used (see [21]) to describe the "backbone exponent" i.e. the case

$j=2$, which is responsible for the dimension of the "backbone" - the sites of percolation cluster connected to the boundary of the domain by two disjoint blue curves - as the leading eigenvalue of a certain differential operator. 
It turns out (as observed by [2]) that the probabilities and the exponents for $j$ blue crossings are different from those for $j$ crossings which are not all of the same colour, and for the latter case there are physical predictions, which we are going to establish. The reason is that one can take two neighboring crossings of different colours, choose their closest (to each other) realizations, and then use their hull as a starting point, choosing "canonical" realizations of other crossings and changing their colours if needed. Similarly to the half-plane case, one concludes that exponents are the same for $j$ crossings of any prescribed colours in any prescribed order, as long as colours are not all the same.

To be more precise, consider critical percolation with fixed mesh equal to 1 , and consider the event $H_{j}(r, R)$ that there exists $j$ disjoint crossings of the annulus $A(r, R):=\{z: r<|z|<R\}$ (in fact we modify $A(r, R)$ as $A_{+}(r, R)$ in the previous section), not all of the same colour. Define $b_{j}(r, R):=\mathbf{P}\left[H_{j}(r, R)\right]$. One can also prescribe colours of the crossings and their order, which will change $b_{j}$ up to a multiplicative constant (we will justify this rigorously later), preserving the theorem below. The goal of this section is to establish the following result, which was predicted by physicists:

Theorem 4 (Plane exponents). For any $j \geq 2$, and for all large enough $r$ (i.e. $r>\operatorname{const}(j))$,

$$
b_{j}(r, R)=R^{-\left(j^{2}-1\right) / 12+o(1)} \text { when } R \rightarrow \infty .
$$

The statement above includes (2) as a particular case: for even $j=2 k$, we can take crossings of alternate colours, and that corresponds to the existence of $k$ disjoint clusters that cross the annulus. In particular, $\mathbf{P}\left(A_{R}^{2}\right)=b_{4}(2, R)=$ $R^{-5 / 4+o(1)}$.

Exactly as its half-plane counterpart, the theorem follows from the two observations which will be discussed below:

- Identification between $S L E_{6}$ and continuum percolation implies that exponents for continuum percolation are equal to the exponents for $S L E_{6}$, computed in [16]. This can be written in terms of the crossings probabilities for the exploration process in the annulus (to be defined below):

$$
\lim _{\rho \rightarrow \infty} b_{j}^{e p}(\rho, R \rho)=R^{-\left(j^{2}-1\right) / 12+o(1)} \text { when } R \rightarrow \infty .
$$

- There is an unbounded set $\mathcal{R} \subset(0, \infty)$ and a positive $c$ (depending on $j$ only) such that crossing probabilities enjoy the following approximate multiplicativity property for any $R \in \mathcal{R}$ and all $n \geq 1$

$$
\begin{aligned}
\operatorname{const}(R) c^{-n} \prod_{l=1}^{n} b_{j}^{e p}\left(2 R^{l}, R^{l+1}\right) & \geq b_{j}\left(R, R^{n+1}\right) \\
& \geq \operatorname{const}(R) c^{n} \prod_{l=1}^{n} b_{j}^{e p}\left(2 R^{l}, 2 R^{l+1}\right) .
\end{aligned}
$$


A more general inequality, analogous to (4) is valid, but for simplicity we prove the above version only, which is sufficient to establish the theorem.

\subsection{Processes in an annulus.}

Exploration process and $S L E_{6}$ in the universal cover. Suppose that an annulus $A=A(r, R)$ is given, and denote by $\tilde{A}=\tilde{A}(r, R)$ its universal cover, with inherited lattice structure. Fix a point $x$ on the outer circle of $A$, let $\tilde{x}$ be one of its lifts to $\tilde{A}$, and $y$ be a "counterclockwise point at infinity on the boundary of $\tilde{A}$."

Perform chordal exploration process and $S L E_{6}$ from $\tilde{x}$ to $y$ in the domain $\tilde{A}$. We define a disconnection time $T$, which is the first time when the projection of the trajectory to the annulus $A$ disconnects the inner circle from the outer one.

As discussed, the law of the chordal exploration process converges (as mesh goes to zero) to that of $S L E_{6}$. If two trajectories of the exploration process are $\delta$-close in the Hausdorff metric, but have drastically different disconnection times, then "six arms" must occur for one of them. The following "a priori bound" (cf. [1, 14])

$$
b_{6}(r, R) \leq \operatorname{const}(r / R)^{-2-\epsilon},
$$

imply that they occur somewhere "at scale $\delta$ " with probability $o(1), \delta \rightarrow 0$. Therefore the law of the chordal exploration process stopped at the disconnection time converges (as mesh goes to zero) to that of $S L E_{6}$ stopped at the disconnection time.

As before, we infer that the probability $b_{j}^{e p}(r, R)$ that the exploration process makes $j-1$ crossings between the inner and outer boundaries of $\tilde{A}$ before time $T$ converges to the similar probability $b_{j}^{s l e}(r, R)$ for $S L E_{6}$ as the mesh of the lattice goes to zero. Increasing the domain while preserving the mesh instead, we conclude that

$$
\lim _{\rho \rightarrow \infty} b_{j}^{e p}(\rho r, \rho R)=b_{j}^{s l e}(r, R) .
$$

Projecting from the universal cover to the annulus. The mentioned chordal processes in $\tilde{A}$ up to the disconnection time $T$ can be projected to the annulus $A$. Locally their definitions coincide with the processes in the annulus described below (which are well-defined up to the disconnection time). So by the restriction property (laws of the exploration process and $S L E_{6}$ depend only on the neighbourhoods of their traces) we conclude that the projections of chordal processes in $\tilde{A}$ coincide with the following processes in $A$ up to the disconnection time $T$ :

- Exploration process in an annulus follows the same "blue to the rightyellow to the left" rule as the chordal exploration process, except that we colour the hexagons of the inner circle in yellow, and that when the exploration process hits the outer circle and the continuous determination of the argument of the exploration process is larger (resp. smaller) than 
that the starting point of the exploration, the boundary point that it hits on the outer circle is blue (resp. yellow).

- $S L E_{6}$ in an annulus goes as radial $S L E_{6}$ (see [16]) from $x$ to the center of the annulus up to the first hitting $\rho$ of the inner boundary. Afterwards it continues like chordal $S L E_{6}$ in the remaining domain, until the disconnection time.

Particularly, we conclude that probabilities $b_{j}^{e p}$ and $b_{j}^{s l e}$ are the same for chordal processes in $\tilde{A}$ and their "annular" counterparts in $A$. Note that scaling implies that just as in the chordal case, $b_{j}^{\text {sle }}(r, R)=: b_{j}^{\text {sle }}(R / r)$.

Exponents for $S L E_{6}$. The computation of exponents for radial and chordal $S L E_{6}$ in $[15,16]$ yields that for $j \geq 2$

$$
b_{j}^{\text {sle }}(R)=R^{-\left(j^{2}-1\right) / 12+o(1)}, \text { when } R \rightarrow \infty .
$$

Indeed, if $\sigma$ now denotes the first hitting time of the inner circle by the $S L E_{6}$ in the annulus $A(1, R)$, and $S / \pi$ the extremal distance between the two circles in $A(1, R) \backslash \gamma[0, \sigma]$, then conformal invariance and the strong Markov property show that

$$
b_{j}^{\text {sle }}(R)=\mathbf{E}\left[1_{\sigma<T} a_{j-2}^{\text {sle }}(S)\right] .
$$

It is shown in [15], Theorem 3.1 that for $\lambda \geq 1$ and $\lambda=0$ (see [3] for this case),

$$
\mathbf{E}\left[1_{\sigma<T} S^{-\lambda}\right] \asymp R^{-\nu(\lambda)} \text { when } R \rightarrow \infty,
$$

where $\nu(\lambda)=(4 \lambda+1+\sqrt{1+24 \lambda}) / 8$. (13) for $j \neq 3$ then follows by plugging in (6). So far, a direct proof of (14) for $\lambda=1 / 3$ is missing in the literature (the proof for $\lambda \geq 1$ in [16] uses the computation of a "derivative exponent" and a convexity argument. The latter does not work directly for $\lambda \in(0,1)$ ). Equation (13) for $j=3$ can however be derived via other rather convoluted means (for instance a universality argument and analyticity of intersection exponents $[18,19])$.

Remark 5. The fact that $b_{2}^{\text {sle }}(R)=R^{-1 / 4+o(1)}$ is related to the fact that the Hausdorff dimension of the $S L E_{6}$ curve is $2-1 / 4=7 / 4$ (see $[25,3]$ ). The fact that $b_{3}^{\text {sle }}(R)=R^{-2 / 3+o(1)}$ is related to the fact that the dimension of the outer frontiers of $S L E_{6}$ and of planar Brownian motion is $2-2 / 3=4 / 3$ (see [19]) and that the fact that $b_{4}^{\text {sle }}(R)=R^{-5 / 4+o(1)}$ is related to the fact that the Hausdorff dimension of the set of (local) cut points of $S L E_{6}$ is $2-5 / 4=3 / 4$ ([16]), e.g. using the simple identification between Brownian hulls and $S L E_{6}$ hulls [32].

Combining (12) and (13) we arrive at (9).

Remark 6. Before proceeding, we want to remark that events corresponding to $b_{j}^{e p}$ can be easily described in terms of percolation crossings. Namely at the time $T$, when exploration process started from the point a first hits the inner circle, its hull $K$ is bounded by two crossings of the annulus: the clockwisemost blue crossing and the counterclockwise-most yellow crossing, containing a between them. After that the exploration process continues as the chordal process 
in $A \backslash K$, creating $(j-2)$ more crossings of alternate colours. Just as in the halfplane case we can change the colours of all crossings except the first two, so we conclude that $b_{j}^{\text {ep }}$ gives (up to a multiplicative constant) the probability of having $(j-2)$ crossings of some prescribed (any) colours outside the hull of two crossings of opposite colours containing a between them.

So, the description of $b_{j}^{e p}$ adds an additional requirement (a prescribed starting point separating two crossings of opposite colour) as compared to $b_{j}$, and we conclude that

$$
b_{j}^{e p}(r, R) \leq b_{j}(r, R)
$$

Unlike the half-plane case, the reverse inequality is valid up to a multiplicative constant only. This is trivial when $j=2 k$ is even (one just has to take alternating colours as prescribed order and then the starting point of the exploration process is anyway between two crossings of different colours) which corresponds to the probability of $k$ disjoint blue clusters. But an additional argument, in the spirit of the discussion below, is needed for odd number of crossings.

4.2. Multiplicativity. Because of the two different colours, the simple argument based on the FKG inequality and the RSW theory can not be immediately applied. Nevertheless, some more elaborate approaches suitable for similar problems were developed by Kesten and others. Needed results for 4 and 5 crossings can be found in [13] and [14] correspondingly, but there seems to be no readily available reference for an arbitrary number of arms, so we present a proof below. Such arguments are also very close to Lawler's separation Lemmas for Brownian intersection probabilities, see e.g., [20].

It follows from [31], that $b_{j}(\rho r, \rho R)$ has a scaling limit, which is conformally invariant, and so depends on the ratio $R / r$ only:

$$
b_{j}^{\prime}(R / r)=\lim _{\rho \rightarrow \infty} b_{j}(\rho r, \rho R) .
$$

By standard RSW theory, $b_{j}(r, R)$ is bounded from below by a power of $R / r$, hence

$$
b_{j}^{\prime}(R) \geq \operatorname{const} R^{-\zeta}
$$

for some $\zeta>0$. Therefore we can conclude that there exist a positive constant $K$ and an unbounded set $\mathcal{R}$ of radii $R$ such that

$$
b_{j}^{\prime}(R / 8) \leq K b_{j}^{\prime}(R / 2) .
$$

We now define a notion of $\delta$-good configurations, when landing points of crossing are "well separated". We say that a configuration is $\delta$-good in the annulus $A\left(r, r^{\prime}\right)$ (we assume that $r^{\prime} \geq 4 r$ ) if there exist $j$ disjoint crossings not all of the same colours, and there is no ball of radius $\delta r$ (resp. $\delta r^{\prime}$ ) centered on the inner (resp. outer) circle and intersecting at least three of the crossings. We call $b_{j}^{\text {good }}\left(r, r^{\prime}\right)=b_{j}^{\text {good }}\left(r, r^{\prime}, \delta\right)$ the probability of this event. Note that the event 
corresponding to $b_{j}\left(r, r^{\prime}\right)-b_{j}^{\text {good }}\left(r, r^{\prime}, \delta\right)$ is contained in the event that there exist $j$ crossings of the annulus $A\left(2 r, r^{\prime} / 2\right)$ and that three crossings of $A(r, 2 r)$ (or of $\left.A\left(r^{\prime} / 2, r^{\prime}\right)\right)$ come $\delta r$-close (resp. $\delta r^{\prime}$ close) near the inner circle (resp. outer circle) of the annulus. From (8) it follows that the probabilities of these last events go to zero when $\delta \rightarrow 0$, (uniformly with respect to $r$ and $r^{\prime}$ ) so we can fix $\delta:=\operatorname{const}(K)$, so that these probabilities are smaller that $1 /(8 K)$ and hence for all $r^{\prime} / r \geq 4$,

$$
b_{j}^{\text {good }}\left(r, r^{\prime}, \delta\right) \geq b_{j}\left(r, r^{\prime}\right)-\frac{1}{4 K} b_{j}\left(2 r, r^{\prime} / 2\right) .
$$

Fix $R \in \mathcal{R}$. It follows from (16) and (17) that there exist $l_{R}:=\operatorname{const}(R)$ such that for $l \geq l_{R}$

$$
b_{j}\left(4 R^{l}, R^{l+1} / 2\right) \leq 2 K b_{j}\left(2 R^{l}, R^{l+1}\right) .
$$

Combining (18) and (19) we conclude that for $l \geq l_{R}$

$$
b_{j}^{g o o d}\left(2 R^{l}, R^{l+1}\right) \geq\left(1-\frac{1}{4 K} 2 K\right) b_{j}\left(2 R^{l}, R^{l+1}\right)=\frac{1}{2} b_{j}\left(2 R^{l}, R^{l+1}\right) .
$$

Standard (but delicate) techniques based on RSW theory (cf. [13]) show that there exists a constant $Q=\operatorname{const}(\delta) \geq 1$ (note that $Q$ depends on $\delta=$ $\operatorname{const}(K)=\operatorname{const}(j))$, such that

$$
b_{j}^{\text {good }}\left(r, r^{\prime}\right) b_{j}^{\text {good }}\left(2 r^{\prime}, r^{\prime \prime}\right) \leq Q b_{j}^{\text {good }}\left(r, r^{\prime \prime}\right) .
$$

Similarly, the exists a constant $Q^{\prime} \geq 1$ such that

$$
b_{j}^{\text {good }}\left(r, r^{\prime} / 2\right) \leq Q^{\prime} b_{j}^{e p}\left(r, r^{\prime}\right) .
$$

We are now ready to conclude, writing for the left half of (10)

$$
\begin{array}{r}
b_{j}\left(R, R^{n+1}\right) \leq \prod_{l=l_{K}}^{n} b_{j}\left(2 R^{l}, R^{l+1}\right) \stackrel{(20)}{\leq} 2^{n} \prod_{l=l_{K}}^{n} b_{j}^{\text {good }}\left(2 R^{l}, R^{l+1}\right) \\
\stackrel{(22)}{\leq} C(R)\left(2 Q^{\prime}\right)^{n} \prod_{l=1}^{n} b_{j}^{e p}\left(2 R^{l}, 2 R^{l+1}\right)
\end{array}
$$

(in the first inequality we used that the event corresponding to $b_{j}\left(R, R^{n+1}\right)$ requires simultaneous occurrence of independent events corresponding to $b_{j}\left(2 R^{l}, R^{l+1}\right)$ with $\left.l=l_{K}, \ldots, n\right)$. For the right half of (10) we write

$$
\begin{aligned}
& b_{j}\left(R, R^{n+1}\right) \geq b_{j}^{\text {good }}\left(R, R^{n+1}\right) \stackrel{(21)}{\geq} C(R) Q^{-n} \prod_{l=l_{K}}^{n} b_{j}^{\text {good }}\left(2 R^{l}, R^{l+1}\right) \\
& \stackrel{(20,15)}{\geq} C(R)(2 Q)^{-n} \prod_{l=1}^{n} b_{j}^{e p}\left(2 R^{l}, R^{l+1}\right) .
\end{aligned}
$$


4.3. One crossing of the annulus. In order to derive (1), one has to translate the existence of one blue connection between circles in terms of the exploration processes. Consider as in the previous subsections a discrete exploration process in the annulus $A(r, R)$ up to its disconnection time $T$ i.e. the first time at which the exploration process contains a closed loop around the inner circle. Let $\rho$ denote the first hitting time of the inner circle.

If $\rho<T$, then it means that there exists one arm of each colour joining the two circles, and in particular a blue one. If $T<\rho$, then one has to see in which direction $\gamma$ did wind around the inner circle: If $\gamma[0, T]$ contains a clockwise loop around the inner circle, then it means that the exploration process has discovered a closed loop of yellow hexagons around the inner circle, and in this case, there is no blue connection between the inner and the outer circle. If however $\gamma[0, T]$ makes an anti-clockwise loop around the inner circle, then the exploration process has discovered a closed loop $l$ of blue hexagons around the inner circle that is connected to the outer circle by a blue path. Furthermore, the exploration process has not explored any of the hexagons that are in the connected component of $A(r, R) \backslash l$ containing the inner circle. Hence, to see if there is a blue crossing of the annulus, it remains to see if there is a blue crossing between the inner circle and $l$ i.e. to start the same algorithm again in this new domain.

Hence, one is lead to study the following quantities: What is the probability that the radial $S L E_{6}$ in the unit disc up to its first hitting time $\rho$ of the circle of radius $r$ contains no clockwise loop around the origin? In [21], this probability is shown to decay like $r^{5 / 48}$. The number $5 / 48$ in fact corresponds to the same differential operator as that describing the probability of no loop at all (i.e. corresponding to the exponent $1 / 4$ ), but with different boundary conditions (one Dirichlet and one Neumann instead of two Dirichlet).

Using arguments in the same spirit than those described above, one can then show that indeed, $\mathbf{P}\left[A_{R}^{1}\right]=R^{-5 / 48+o(1)}$. For details, we refer to [21]. Note that this is equivalent to

$$
\mathbf{P}[0 \text { is connected to } x]=|x|^{-5 / 24+o(1)}
$$

when $x$ goes to infinity (i.e., the exponent often denoted by $\eta$ exists and is equal to $5 / 24)$.

\section{Some open questions}

To conclude, we very briefly list some questions that seem still open at this moment. Some of them are probably within reach, and some are less accessible.

1. Generalizing the results of Smirnov [30], to other lattices. The first two natural candidates are bond percolation on the square lattice and site percolation on a Voronoi tesselation (see e.g. [4]), that both have a "selfduality" type property (in particular, the value $p=1 / 2$ has to be studied). It would be sufficient to prove Cardy's formula, but in both cases the method used in [30] does not apply directly. 
2. Existence of two, three, and four arms from the vicinity of a site represent it belonging to frontier of a percolation cluster, perimeter of a percolation cluster, and being a pivotal site respectively. Thus we infer that on a lattice with mesh 1 , when we speak of clusters of size $\approx N$, a site has probablity $\approx N^{-1 / 4}$ to belong to a frontier, probability $\approx N^{-2 / 3}$ to belong to perimeter, and probability $\approx N^{-5 / 4}$ of being pivotal. One should be able to show a stronger statement, roughly speaking that a cluster of size $\approx N$ has frontier of $\approx N^{2-1 / 4}=N^{7 / 4}$ sites, perimeter of $\approx N^{2-2 / 3}=N^{4 / 3}$ sites, and $\approx N^{2-5 / 4}=N^{3 / 4}$ pivotal sites. The counterpart of this stronger claim for continuum percolation follows from the identification between $S L E_{6}$ and the scaling limit of percolation cluster perimeter: e.g., the scaling limit of the frontier of a percolation cluster has the same law (when properly normalized) as the Brownian frontier, and hence has Hausdorff dimension $4 / 3$ almost surely. See [3] for a more direct proof.

3. Show that the power laws hold up to constants. For instance, does $\mathbf{P}\left[A_{R}^{1}\right] \in$ $\left[c R^{-5 / 48}, C R^{-5 / 48}\right]$ hold for some constants $c, C \in(0, \infty)$ ? Estimates up to constants can be useful in order to derive results on "discrete fractal dimension" (see the previous question).

4. Show that the exponent $\alpha$ associated to the mean number of clusters per vertex exists and determine its value (conjectured to be $-2 / 3$ ).

5. Determine the exponents corresponding to $j \geq 3$ blue crossings of an annulus.

6. Understand the relation between other critical lattice models such as the critical random cluster models and the relation to other $S L E_{\kappa}$ and their critical exponents.

\section{Acknowledgements}

We thank Raphael Cerf and Oded Schramm for valuable comments and Harry Kesten for very useful advice.

\section{References}

[1] M. Aizenman (1996), The geometry of critical percolation and conformal invariance, in STATPHYS 19 (Xiamen, 1995), 104-120. World Sci. Publishing, River Edge, NJ, 1996.

[2] M. Aizenman, B. Duplantier, A. Aharony (1999), Path crossing exponents and the external perimeter in 2D percolation, Phys. Rev. Let. 83, 1359-1362.

[3] V. Beffara (2001), in preparation.

[4] I. Benjamini, O. Schramm (1998), Conformal invariance of Voronoi percolation, Comm. Math. Phys. 197 (1998), no. 1, 75-107.

[5] J.L. Cardy (1984), Conformal invariance and surface critical behaviour, Nucl. Phys. B240, 514-532.

[6] J.L. Cardy (1998), The number of incipient spanning clusters in two-dimensional percolation, J. Phys. A 31, L105.

[7] J.T. Chayes, L. Chayes, J. Fröhlich, The low-temperature behavior of disordered magnets, Comm. Math. Phys. 100, (1985), no. 3, 399-437.

[8] M.P.M. Den Nijs (1979), A relation between the temperature exponents of the eight-vertex and the q-state Potts model, J. Phys. A 12, 1857-1868. 
[9] B. Duplantier (1999), Harmonic measure exponents for two-dimensional percolation, Phys. Rev. Lett. 82, 3940-3943.

[10] G. Grimmett (1999), Percolation. Second Edition. Springer-Verlag, Berlin, 1999.

[11] T. Grossman, A. Aharony (1987), Accessible external perimeters of percolation clusters, J.Physics A 20, L1193-L1201.

[12] H. Kesten (1982), Percolation theory for mathematicians, Progress in Probability and Statistics, 2. Birkhäuser, Boston, 1982.

[13] H. Kesten, Scaling relations for 2D-percolation, Comm. Math. Phys. 109, (1987), no. 1, 109-156.

[14] H. Kesten, V. Sidoravicius, Y. Zhang (1998), Almost all words are seen in critical site percolation on the triangular lattice, Electron. J. Probab. 3, no. 10, 75pp.

[15] G.F. Lawler, O. Schramm, W. Werner (1999), Values of Brownian intersection exponents I: Half-plane exponents, Acta Mathematica, to appear, arXiv:math.PR/9911084.

[16] G.F. Lawler, O. Schramm, W. Werner (2000), Values of Brownian intersection exponents II: Plane exponents, Acta Mathematica, to appear, arXiv:math.PR/0003156.

[17] G.F. Lawler, O. Schramm, W. Werner (2000), Values of Brownian intersection exponents III: Two sided exponents, Ann. Inst. Henri Poincaré, to appear, arXiv:math.PR/0005294.

[18] G.F. Lawler, O. Schramm, W. Werner (2000), Analyticity of intersection exponents for planar Brownian motion, Acta Mathematica, to appear, arXiv:math.PR/0005295.

[19] G.F. Lawler, O. Schramm, W. Werner (2001), The dimension of the planar Brownian frontier is 4/3, Math. Res. Lett. 8, no. 4, 401-411.

[20] G.F. Lawler, O. Schramm, W. Werner (2001), Sharp estimates for Brownian non-intersection probabilities, in "In and out of equilibrium, probability with a physics flavour," Progress in Probability and Statistics, Birkhäuser, to appear, arXiv:math.PR/0101247.

[21] G.F. Lawler, O. Schramm, W. Werner (2001), One-arm exponent for $2 D$ critical percolation, to appear in the Electronic Journal of Probability, arXiv:math.PR/0108211.

[22] B. Nienhuis, E.K. Riedel, M. Schick (1980), Magnetic exponents of the two-dimensional q-states Potts model, J. Phys A 13, L. 189-192.

[23] B. Nienhuis, Critical behavior of two-dimensional spin models and charge asymmetry in the Coulomb gas, J. Statist. Phys. 34 (1984), no. 5-6, 731-761.

[24] R.P. Pearson (1980), Conjecture for the extended Potts model magnetic eigenvalue, Phys. Rev. B 22, 2579-2580.

[25] S. Rohde, O. Schramm (2001), Basic properties of SLE, preprint, arXiv:math.PR/0106036.

[26] H. Saleur, B. Duplantier (1987), Exact determination of the percolation hull exponent in two dimensions, Phys. Rev. Lett. 58, no. 22, 2325-2328.

[27] B. Sapoval, M. Rosso, J.F. Gouyet (1985), The fractal nature of a diffusion front and the relation to percolation, J. Physique Lett. 46, L149-L156.

[28] O. Schramm (2000), Scaling limits of loop-erased random walks and uniform spanning trees, Israel J. Math. 118, 221-288.

[29] O. Schramm (2001), A percolation formula, preprint, arXiv:math.PR/0107096.

[30] S. Smirnov (2001), Critical percolation in the plane: Conformal invariance, Cardy's formula, scaling limits, C. R. Acad. Sci. Paris Sr. I Math. 333 (2001), no. 3, 239-244.

[31] S. Smirnov (2001), in preparation.

[32] W. Werner (2000), Critical exponents, conformal invariance and planar Brownian motion, Proc. 3ECM 2000, Birkhäuser, to appear, arXiv:math.PR/0007042.

[33] Y. Zhang (2001), in preparation. 
Department of Mathematics, Royal Institute of Technology (KTH), S-10044 StockHOLM, SWEDEN.

E-mail address: stas@math.kth.se

Département de Mathématiques, BÂt. 425, Université Paris-Sud, F-91405 Orsay CEDEX, France.

E-mail address: wendelin.werner@math.u-psud.fr 